\title{
Sparse whole-genome sequencing identifies two loci for major depressive disorder
}

\author{
CONVERGE consortium*
}

\begin{abstract}
Major depressive disorder (MDD), one of the most frequently encountered forms of mental illness and a leading cause of disability worldwide ${ }^{1}$, poses a major challenge to genetic analysis. To date, no robustly replicated genetic loci have been identified ${ }^{2}$, despite analysis of more than 9,000 cases $^{3}$. Here, using low-coverage whole-genome sequencing of 5,303 Chinese women with recurrent MDD selected to reduce phenotypic heterogeneity, and 5,337 controls screened to exclude MDD, we identified, and subsequently replicated in an independent sample, two loci contributing to risk of MDD on chromosome 10: one near the SIRT1 gene $\left(P=2.53 \times 10^{-10}\right)$, the other in an intron of the LHPP gene $\left(P=6.45 \times 10^{-12}\right)$. Analysis of 4,509 cases with a severe subtype of MDD, melancholia, yielded an increased genetic signal at the SIRT1 locus. We attribute our success to the recruitment of relatively homogeneous cases with severe illness.
\end{abstract}

The existence and number of subtypes of depression have been debated over the past 100 years. The current consensus is that depression may be a collection of partly distinct diseases, with overlapping causal pathways. This aetiologic heterogeneity might therefore substantially reduce the power of genetic association studies, and hence explain the failure to find genetic risk loci $^{3}$. For example, there may be cases of MDD of largely environmental origin whose presence reduces the power to detect genetic effects. Also, genetic risk factors for mild depressive syndromes may not be entirely the same as those for more severe cases ${ }^{4}$.

For these reasons, we investigated the genetic basis of MDD in subjects for whom known sources of phenotypic and genetic heterogeneity were minimized and known risk factors documented. The CONVERGE (China, Oxford and Virginia Commonwealth University Experimental Research on Genetic Epidemiology) consortium recruited 11,670 Han Chinese women through a collaboration involving 58 hospitals in China. We studied only women because about $45 \%$ of the genetic liability to MDD is not shared between sexes ${ }^{5,6}$. In an attempt to obtain severe cases of MDD, we recruited only recurrent cases (mean number of episodes was 5.6).

We used low-coverage sequencing to genotype our sample ${ }^{7}$. Wholegenome sequences were acquired to a mean depth of $1.7 \times(95 \%$ confidence intervals (CIs) 0.7-4.3) per individual, from which 32,781,340 SNP sites were identified. After applying stringent quality controls (Methods), we obtained 10,640 samples (5,303 cases of MDD, 5,337 controls) and 6,242,619 SNPs for inclusion in genome-wide association studies (GWAS). We compared genotypes from the low-coverage sequencing to genotypes called with $10 \times$ coverage sequence and to genotypes called from genotyping arrays and a mass spectrometer platform. The mean percentage concordance between genotypes from nine individuals with both low- and $10 \times$ coverage across all sites was 98.1\% (Supplementary Table 1). We compared imputed genotypes to those acquired for 72 individuals using an array and to 21 SNPs genotyped on all individuals with the MassARRAY system mass spectrometer (Supplementary Notes). Overall concordance was 98.0\% (Supplementary Tables 2 and 3).
Genetic association analysis was carried out with a linear mixed model with a genetic relatedness matrix (GRM) as a random effect and principal components from eigen-decomposition of the GRM as fixed effect covariates (Methods, Supplementary Notes) ${ }^{8,9}$. Fig. 1a and Extended Data Fig. 1 show the Manhattan and quantile-quantile plots, respectively, for this analysis. The genomic control inflation factor $(\lambda$, the ratio of the observed median $\chi^{2}$ to that expected by chance) for association with MDD was 1.070 (for common SNPs, minor allele frequency (MAF) $>2 \%, \lambda=1.074)$. The adjusted measure for sample size to that of 1,000 cases and 1,000 controls $\left(\lambda_{1000}\right)$ was 1.013 .

Two loci exceeded genome-wide significance in association with MDD: one $5^{\prime}$ to the sirtuin1 (SIRT1) gene on chromosome 10 (SNP = rs12415800, chromosome 10:69624180, $\mathrm{MAF}=45.2 \%, P=1.92 \times 10^{-8}$, Fig. 1b), and the other in an intron of the phospholysine phosphohistidine inorganic pyrophosphate phosphatase (LHPP) gene $(\mathrm{SNP}=\mathrm{rs35936514}$, chromosome 10:126244970, $\mathrm{MAF}=26.0 \%$, $P=1.27 \times 10^{-8}$, Fig. 1c). All SNPs with $P$ values of association $<10^{-5}$ with MDD are listed in Supplementary Table 4.

We checked the accuracy of the imputed genotypes at 12 SNPs with $P<1 \times 10^{-5}$, by re-genotyping the CONVERGE samples using a MassARRAY system mass spectrometer, thereby confirming their association with MDD. Extended Data Table 1 shows that the correlation between the two assays was high (mean $r^{2}=0.984$ ), and the odds ratios for the two genome-wide significant SNPs assessed by the two methods were almost identical, with highly overlapping confidence intervals (rs12415800 odds ratios: 1.167 versus 1.167 ; rs35936514 odds ratios: 0.845 versus 0.842 ).

We replicated the associations by genotyping the same 12 SNPs in a separate Han Chinese cohort of 3,231 cases with recurrent MDD, and 3,186 controls (both sexes). Two SNPs at the peaks of association for SIRT1 and LHPP loci (rs12415800 and rs35936514, respectively) for MDD in the CONVERGE samples were significantly associated with MDD (Table 1). Analysis of the combined samples gave $P$ values for association with MDD at these two SNPs of $2.53 \times 10^{-10}$ and $6.45 \times 10^{-12}$, respectively. Extended Data Table 2 shows the genotype distribution and $P$ values for tests of violation of the Hardy-Weinberg equilibrium in both the CONVERGE samples and the replication cohort at both SNPs.

Comparison with results from the Psychiatric Genomics Consortium (PGC) mega-analysis of European studies ${ }^{3}$ failed to provide robust replication for our top SNPs (Extended Data Fig. 2 and Extended Data Table 3). However, the proportion of associations in the same direction in the two studies exceeded expectations due to chance $(P<0.001)$, and polygenic risk scores from the PGC megaanalysis applied to the CONVERGE samples were of significant $(P<0.01)$ but limited predictive value, accounting for $0.1 \%$ of MDD risk in the CONVERGE cohort (Extended Data Table 4). It is unclear to what extent differences in sample ascertainment, ethnicity, or other factors contribute to the failure to replicate genetic effects in the PGC sample. Notably, variants at our most strongly associated loci are much rarer in European populations, where rs12415800 (SIRT1) 

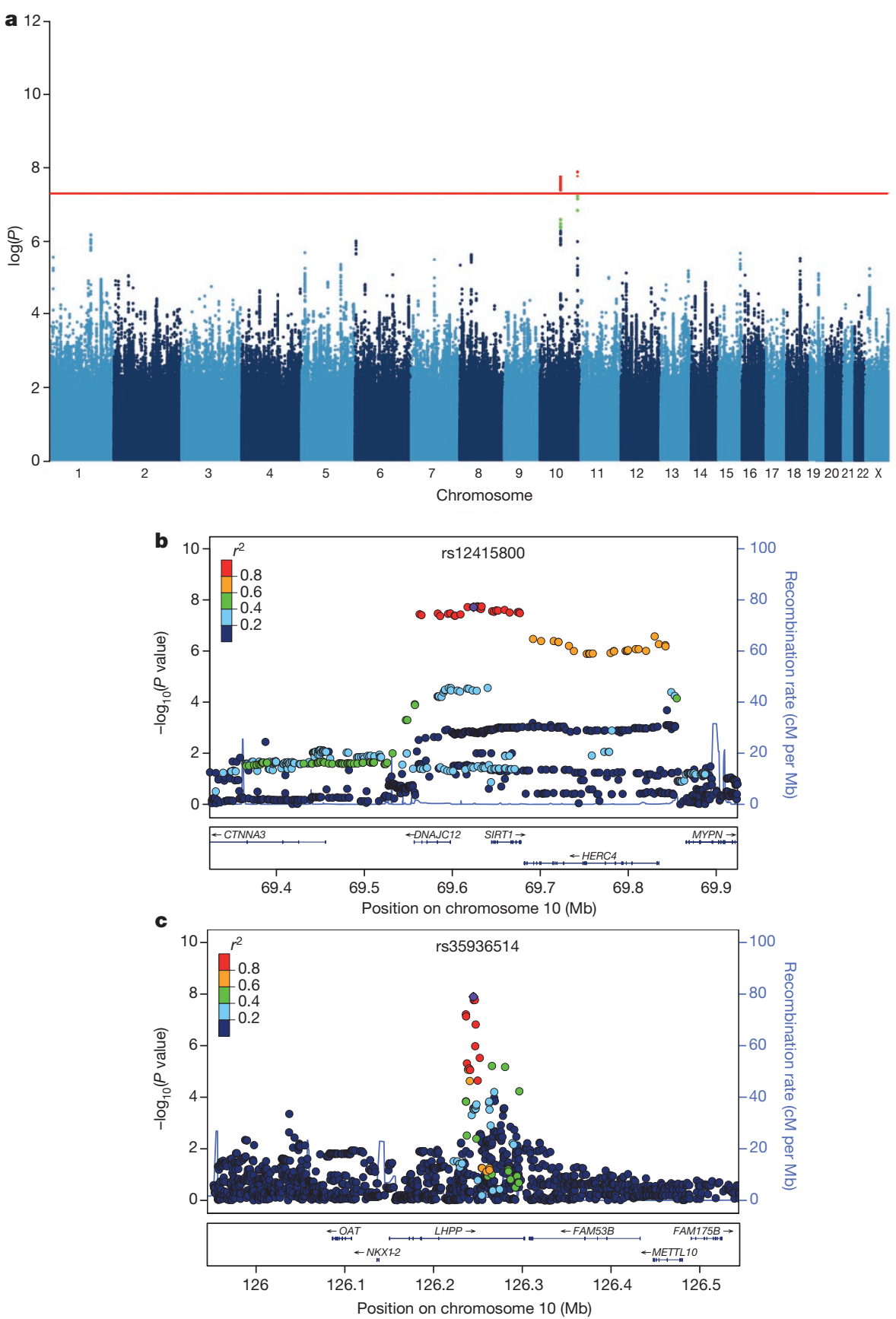

Figure $1 \mid$ Two loci associated with MDD in the CONVERGE sample. a, Manhattan plot of genome-wide association for MDD. b, Association at the SIRT1 region on chromosome 10 at 69.6 megabases (Mb). c, Association at the LHPP gene on chromosome 10 at position $126.2 \mathrm{Mb}$. For b and $\mathbf{c}$ The $-\log _{10}(P$ value $)$ of imputed SNPs associated with MDD is shown on the left $y$ axis. The recombination rates expressed in centimorgans (cM) per Mb (NCBI Build GRCh37; light blue lines), are shown on the right $y$ axis. Position in $\mathrm{Mb}$ is on the $x$ axis. Linkage disequilibrium of each SNP with the top SNP, displayed as a large purple diamond, is indicated by its colour. The plots were drawn using LocusZoom ${ }^{20}$. and rs 35936514 (LHPP) have frequencies of $3 \%$ and $8 \%$ respectively, compared to $45 \%$ and $26 \%$ in the CONVERGE cohort.

We considered whether successful mapping of MDD in the CONVERGE samples was attributable to the recruitment of a severe, more genetically determined form of the disease. We tested that hypothesis by looking within the CONVERGE cohort at a particularly severe, and more heritable form of MDD: melancholia ${ }^{10}$. Prior research has suggested that MDD patients with melancholia have more impairing, recurrent episodes and that risk for MDD is higher in the co-twins of probands with the melancholic subtype ${ }^{11}$ than in those with non-melancholic MDD. This increase is greater in monozygotic than dizygotic twin pairs ${ }^{11}$, as would be expected if the subtype were associated with greater genetic risk.

In the CONVERGE cohort, $85 \%$ of cases met the DSM-IV criteria for melancholia ${ }^{12}$. We searched for a genetic association in 9,846 samples (4,509 cases and 5,337 controls) and identified the same two loci that exceeded genome-wide significance on chromosome 10 . The genomic control inflation factor $\lambda$ for melancholia was 1.069 , and $\lambda_{1000}$ was 1.014 . Even though the sample for melancholia was smaller than for MDD, at the SIRT1 locus the significance of association was two orders of magnitude greater than for MDD (top SNP = rs80309727, chromosome 10:69617347, $\mathrm{MAF}=45.2 \%, P=2.95 \times$ $\left.10^{-10}\right)$. Extended Data Fig. 3 shows the Manhattan plot, quantilequantile plot and detailed views of the SIRT1 locus associated with melancholia. All SNPs with $P$ values of association $<10^{-5}$ with melancholia are listed in Supplementary Table 5. To determine whether the increased association might have arisen by chance, we generated an empirical distribution of odds ratios by randomly selecting 4,509 cases from the total set and re-analysing the association with each of the genome-wide significant variants. We found that the observed value lay on the 98.8th percentile at the SIRT1 locus, but at the $61.6^{\text {th }}$ percentile at the LHPP locus (Extended Data Fig. 4).

Our results indicate that, as others have suggested ${ }^{13}$, obtaining lowsequence coverage of a large number of individuals can be an effective 
Table 1 | Genetic association between MDD and 12 variants in the CONVERGE cohort and a replication sample

\begin{tabular}{|c|c|c|c|c|c|c|c|c|c|c|c|c|c|c|c|}
\hline \multicolumn{5}{|c|}{ SNP } & \multicolumn{5}{|c|}{ CONVERGE $(n=10,640)$} & \multicolumn{3}{|c|}{ Replication $(n=6,417)$} & \multicolumn{3}{|c|}{ Joint $(n=17,057)$} \\
\hline Chr. & Pos. & RSID & Ref. & Alt. & Freq. & Info. & OR & s.e. & $P$ & OR & s.e. & $P$ & OR & s.e. & $P$ \\
\hline 1 & 11493832 & rs2922240 & $\mathrm{T}$ & C & 0.385 & 1.018 & 1.141 & 0.028 & $2.80 \times 10^{-6}$ & 0.949 & 0.037 & $1.54 \times 10^{-1}$ & 1.070 & 0.022 & $2.46 \times 10^{-3}$ \\
\hline 1 & & 3766688 & $\mathrm{~T}$ & C & 0.394 & 1.003 & 0.8 & 0.0 & $1.83 \times 10^{-6}$ & 0.991 & 0.037 & $8.15 \times 10^{-1}$ & 0.918 & .022 & $1.34 \times 10^{-4}$ \\
\hline 1 & 228052027 & rs57047840 & A & G & 0.284 & 0.970 & 1.138 & 0.031 & $4.64 \times 10^{-5}$ & 1.001 & 0.041 & $9.90 \times 10^{-1}$ & 1.088 & 0.025 & $5.57 \times 10^{-4}$ \\
\hline 5 & 9161674 & rs55713588 & A & G & 0.096 & 0.893 & 1.278 & 0.050 & $6.04 \times 10^{-7}$ & 1.054 & 0.062 & $3.93 \times 10^{-1}$ & 1.042 & 0.035 & $2.08 \times 10^{-1}$ \\
\hline 6 & 4386107 & rs55800092 & C & $\mathrm{T}$ & 0.151 & 1.001 & 0.824 & 0.039 & $1.35 \times 10^{-6}$ & 0.962 & 0.052 & $4.49 \times 10^{-1}$ & 0.876 & 0.031 & $1.82 \times 10^{-5}$ \\
\hline 10 & 69624180 & rs12415800 & G & A & 0.452 & 0.992 & 1.164 & 0.028 & $1.92 \times 10^{-8}$ & 1.130 & 0.036 & $7.71 \times 10^{-4}$ & 1.150 & 0.022 & $2.37 \times 10^{-10}$ \\
\hline 10 & 126244970 & rs35936514 & C & $T$ & 0.260 & 0.993 & 0.839 & 0.032 & $1.27 \times 10^{-8}$ & 0.838 & 0.041 & $1.68 \times 10^{-5}$ & 0.842 & 0.025 & $6.43 \times 10^{-12}$ \\
\hline 13 & 107659212 & rs61967003 & C & $\mathrm{T}$ & 0.017 & 0.999 & 1.645 & 0.109 & $6.70 \times 10^{-6}$ & 0.788 & 0.150 & $1.11 \times 10^{-1}$ & 1.277 & 0.087 & $4.81 \times 10^{-3}$ \\
\hline 14 & 66833851 & rs17827252 & C & G & 0.463 & 1.011 & 0.887 & 0.028 & $1.44 \times 10^{-5}$ & 0.962 & 0.041 & $3.41 \times 10^{-1}$ & 0.907 & 0.023 & $2.20 \times 10^{-5}$ \\
\hline 19 & 34493757 & rs11880240 & C & G & 0.068 & 1.019 & 1.291 & 0.055 & $8.02 \times 10^{-6}$ & 1.048 & 0.072 & $5.12 \times 10^{-1}$ & 1.184 & 0.043 & $9.15 \times 10^{-5}$ \\
\hline$x$ & 24656658 & & A & G & 0.721 & 0.995 & 0.883 & 0.031 & $3.22 \times 10^{-5}$ & 0.994 & 0.047 & $9.01 \times 10^{-1}$ & 0.917 & 0.026 & $1.09 \times 10^{-3}$ \\
\hline$x$ & 25011374 & rs11573525 & C & $\mathrm{T}$ & 0.260 & 0.971 & 1.160 & 0.032 & $5.86 \times 10^{-6}$ & 1.011 & 0.047 & $8.19 \times 10^{-1}$ & 1.100 & 0.027 & $2.18 \times 10^{-4}$ \\
\hline
\end{tabular}

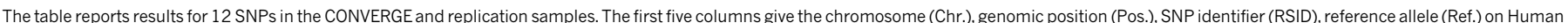

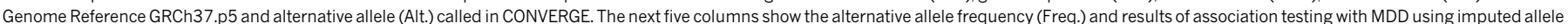

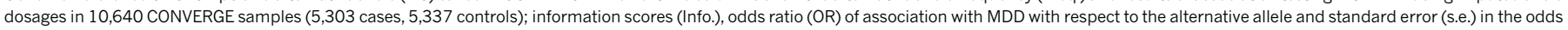
ratio were obtained from a logistic regression model; $P$ values of association $(P)$ were obtained from a linear-mixed model with a GRM containing all samples. The next three columns present the results of

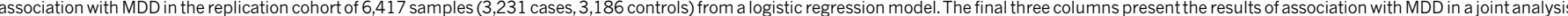
with both CONVERGE and replication cohorts from a logistic regression model. Bold type indicates the genome-wide significant markers.

way to screen the genome for association signals. We were able to genotype more variants than on genotyping arrays and our set is larger than publicly available sources for imputation ${ }^{14}$. Our imputation pipeline employed standard tools, and it is likely that imputation accuracy could be improved with further algorithmic research.

MDD is most probably highly polygenic ${ }^{3}$, and many additional loci remain to be discovered. We attribute the discovery and replication of two SNPs associated with MDD in the CONVERGE cohort to the recruitment of cases who were probably more homogeneous and more severely impaired than those collected in previous studies from Western cultures. In East Asia, reluctance to report $\mathrm{MDD}^{15}$ probably explains why hospital-ascertained cases are more severe, and why prevalence estimates for MDD are lower in China $\left(3.6 \%{ }^{16}\right)$ than in the US $(16.2 \%)^{17}$. Consistent with this interpretation, $85 \%$ of the cases of MDD in the CONVERGE cohort have melancholia, a severe subtype of MDD; mapping melancholia led to a significant increase in the genetic signal at one locus. Finally, we note that one of the replicated risk loci is located close to a gene involved in mitochondrial biogenesis $(S I R T 1)^{18}$, which, together with our finding that MDD is associated with increased amounts of mitochondrial DNA ${ }^{19}$, suggests an unexpected origin for at least some of the phenotypic manifestations of MDD.

Online Content Methods, along with any additional Extended Data display items and Source Data, are available in the online version of the paper; references unique to these sections appear only in the online paper.

Received 15 December 2014; accepted 11 June 2015.

Published online 15 July 2015.

1. Kessler, R. C. \& Bromet, E. J. The epidemiology of depression across cultures. Annu Rev. Public Health 34, 119-138 (2013).

2. Flint, J. \& Kendler, K. S. The genetics of major depression. Neuron $\mathbf{8 1 , 4 8 4 - 5 0 3}$ (2014).

3. Major Depressive Disorder Working Group of the Psychiatric GWAS Consortium et al. A mega-analysis of genome-wide association studies for major depressive disorder. Mol. Psychiatry 18, 497-511 (2013).

4. Foley, D. L. etal. Genetic and environmental risk factors for depression assessed by subject-rated symptom check list versus structured clinical interview. Psychol. Med. 31, 1413-1423 (2001).

5. Kendler, K. S. et al. Clinical indices of familial depression in the Swedish Twin Registry. Acta Psychiatr. Scand. 115, 214-220 (2007).

6. Sullivan, P. F. et al. Genetic epidemiology of major depression: review and metaanalysis. Am. J. Psychiatry 157, 1552-1562 (2000).

7. Li, Y. et al. Low-coverage sequencing: implications for design of complex trait association studies. Genome Res. 21, 940-951 (2011).

8. Lippert, C. et al. FaST linear mixed models for genome-wide association studies. Nature Methods 8, 833-835 (2011)

9. Widmer, C. et al. Further improvements to linear mixed models for genome-wide association studies. Sci. Rep. 4, 6874 (2014).

10. Angst, J. et al. Melancholia and atypical depression in the Zurich study: epidemiology, clinical characteristics, course, comorbidity and personality. Acta Psychiatr. Scand. Suppl. 433, 72-84 (2007).
11. Kendler, K. S. The diagnostic validity of melancholic major depression in a population-based sample of female twins. Arch. Gen. Psychiatry 54, 299-304 (1997).

12. Sun, N. et al. A comparison of melancholic and nonmelancholic recurrent major depression in Han Chinese women. Depress. Anxiety 29, 4-9 (2012).

13. Pasaniuc, B. et al. Extremely low-coverage sequencing and imputation increases power for genome-wide association studies. Nature Genet. 44, 631-635 (2012).

14. Abecasis, G. R. et al. An integrated map of genetic variation from 1,092 human genomes. Nature 491, 56-65 (2012).

15. Liao, S. C. et al. Low prevalence of major depressive disorder in Taiwanese adults: possible explanations and implications. Psychol. Med. 42, 1227-1237 (2012).

16. Lee, S. et al. The epidemiology of depression in metropolitan China. Psychol. Med. 39, 735-747 (2009).

17. Kessler, R. C. etal. The epidemiology of major depressive disorder: results from the National Comorbidity Survey Replication (NCS-R). J. Am. Med. Assoc. 289, 3095-3105 (2003).

18. Gerhart-Hines, Z et al. Metabolic control of muscle mitochondrial function and fatty acid oxidation through SIRT1/PGC-1 $\alpha$. EMBO J. 26, 1913-1923 (2007).

19. Cai, N. et al. Molecular signatures of major depression. Curr. Biol. 25, 1146-1156 (2015).

20. Pruim, R. J. et al. LocusZoom: regional visualization of genome-wide association scan results. Bioinformatics 26, 2336-2337 (2010).

Supplementary Information is available in the online version of the paper.

Acknowledgements This work was funded by the Wellcome Trust (WT090532/Z/09/ Z, WT083573/Z/07/Z, WT089269/Z/09/Z), NIH grant MH-100549 and the Brain and Behavior Research Foundation. All authors are part of the CONVERGE consortium (China, Oxford and VCU Experimental Research on Genetic Epidemiology) and gratefully acknowledge the support of all partners in hospitals across China. W. Kretzschmar is funded by the Wellcome Trust (WT097307). N. Cai is supported by the Agency of Science, Technology and Research (A*STAR) Graduate Academy. J. Marchini is funded by an ERC Consolidator Grant (617306). Q. Xu is funded by the 973 Program (2013CB531301) and NSFC (31430048, 31222031).

Author Contributions Manuscript preparation: N. Cai, T. B. Bigdeli, W. Kretzschmar, M. Reimers, T. Webb, B. Riley, S. Bacanu, R. E. Peterson, K. S. Kendler and J. Flint. Replication sample: O. Xu CONVERGE sample collection: Yih. Li, Y. Chen, H. Deng W. Sang, Ke. Li, J. Gao, B. Ha, S. Gao, J. Hu, C. Hu, G. Huang, G. Jiang, X. Zhou, You. Li, Kan Li, Q. Niu, Yi Li, G. Li, L. Liu, Z. Liu, Yi Li, X. Fang, R. Pan, G. Miao, Q. Zhang, F. Yu, G. Chen, M. Cai, D. Yang, X. Hong, Y. Song, C. Gao, J. Pan, Y. Zhang, T. Liu, J. Dong, X. Wang, L. Wang, Q. Mei, Z. Shen, X. Liu, W. Wu, D. Gu, Y. Chen, T. Liu, H. Rong, Yi. Liu, L. Lv, H. Meng, H. Sang, J. Shen, T. Tian, J. Shi, J. Sun, M. Tao, X. Wang, J. Xia, Q. He, G. Wang, X. Wang, Lina Yang, K. Zhang, N. Sun, J. Zhang, Z. Gan, Z. Zhang, W. Zhang, H. Zhong, F. Yang, E. Cong, S. Shi, G. Fu, J. Flint and K. S. Kendler. Genome sequencing and analysis: J. Liang, J. Hu, Q. Li, W. Jin, Z. Hu, G. Wang, Linm. Wang, P. Qian, Yu. Liu, T. Jiang, Y. Lu, X. Zhang, Y. Yin, Yin. Li, H. Yang, Jia. Wang, X. Gan, Yih. Li, N. Cai, R. Mott, J. Flint, Jun Wang and X. Xu. Genotype imputation: W. Kretzschmar, J. Hu, L. Song, Q. Li, N. Cai and J. Marchini. Genetic analysis: N. Cai, T. Bigdeli, Yih. Li, R. E. Peterson, S. Bacanu, T. Webb, B. Riley, K. S. Kendler, R. Mott and J. Flint.

Author Information All sequence data and MDD results are freely available at http:// dx.doi.org/10.5524/100155. GWAS results are also available at http:// www.med.unc.edu/pgc/downloads. Reprints and permissions information is available at www.nature.com/reprints. The authors declare no competing financial interests. Readers are welcome to comment on the online version of the paper. Correspondence and requests for materials should be addressed to Q. Xu (xuqi@pumc.edu.cn), Jun Wang (wangj@genomics.org.cn), K. S. Kendler (kendler@vcu.edu) or J. Flint (jf@well.ox.ac.uk). 


\section{CONVERGE consortium}

Na Cai ${ }^{1 *}$, Tim B. Bigdeli ${ }^{2 *}$, Warren Kretzschmar ${ }^{1 *}$, Yihan Li ${ }^{1 *}$, Jieqin Liang ${ }^{3}$, Li Song ${ }^{3}$, Jingchu $\mathrm{Hu}^{3}$, Qibin $\mathrm{Li}^{3}$, Wei Jin ${ }^{3}$, Zhenfei $\mathrm{Hu}^{3}$, Guangbiao Wang ${ }^{3}$, Linmao Wang ${ }^{3}$, Puyi Qian ${ }^{3}$, Yuan Liu ${ }^{3}$, Tao Jiang ${ }^{3}$, Yao Lu ${ }^{3}$, Xiuqing Zhang ${ }^{3}$, Ye Yin $^{3}$, Yingrui Li ${ }^{3}$, Xun Xu $^{3}$, Jingfang Gao ${ }^{4}$, Mark Reimers ${ }^{2}$, Todd Webb², Brien Riley², Silviu Bacanu ${ }^{2}$, Roseann E. Peterson ${ }^{2}$, Yiping Chen ${ }^{5}$, Hui Zhong ${ }^{6}$, Zhengrong Liu', Gang Wang ${ }^{8}$, Jing Sun ${ }^{9}$, Hong Sang ${ }^{10}$, Guoqing Jiang ${ }^{11}$, Xiaoyan Zhou ${ }^{11}$, Yi Li ${ }^{12}, Y_{i}$ Li $^{13}$, Wei Zhang ${ }^{14}$ Xueyi Wang ${ }^{15}$, Xiang Fang ${ }^{16}$, Runde Pan ${ }^{17}$, Guodong Miao ${ }^{18}$, Qiwen Zhang ${ }^{19}$, Jian Hu ${ }^{20}$ Fengyu $\mathrm{Yu}^{21}$, Bo Du ${ }^{22}$, Wenhua Sang ${ }^{22}$, Keqing Li ${ }^{22}$, Guibing Chen ${ }^{23}$, Min Cai ${ }^{24}$, Lijun Yang ${ }^{25}$, Donglin Yang ${ }^{26}$, Baowei Ha ${ }^{27}$, Xiaohong Hong ${ }^{28}$, Hong Deng ${ }^{29}$, Gongying Li ${ }^{30}$, Kan Li ${ }^{31}$, Yan Song ${ }^{32}$, Shugui Gao ${ }^{33}$, Jinbei Zhang ${ }^{34}$, Zhaoyu 'Gan ${ }^{34}$, Huaqing Meng ${ }^{35}$, Jiyang Pan ${ }^{36}$, Chengge Gao ${ }^{37}$, Kerang Zhang ${ }^{38}$, Ning Sun ${ }^{38}$, Youhui $\mathrm{Li}^{39}$, Qihui $\mathrm{Niu}^{39}$, Yutang Zhang ${ }^{40}$, Tieqiao Liu ${ }^{41}$, Chunmei Hu ${ }^{42}$, Zhen Żhang ${ }^{43}$, Luxian Lv ${ }^{44}$, Jicheng Dong ${ }^{45}$, Xiaoping Wang ${ }^{46}$, Ming Tao ${ }^{47}$, Xumei Wang ${ }^{48}$, Jing Xia ${ }^{48}$ Han Rong ${ }^{49}$, Qiang He $\mathrm{H}^{50}$, Tiebang Liu ${ }^{51}$, Guoping Huang ${ }^{52}$, Qiyi Mei ${ }^{53}$, Zhenming Shen ${ }^{54}$, Ying Liu ${ }^{55}$, Jianhua Shen ${ }^{56}$, Tian Tian ${ }^{56}$, Xiaojuan Liu ${ }^{57}$, Wenyuan Wu ${ }^{58}$, Danhua Gu ${ }^{59}$, Guangyi Fu ${ }^{1}$, Jianguo Shi ${ }^{60}$, Yunchun Chen ${ }^{61}$, Xiangchao Gan ${ }^{62}$, Lanfen Liu $^{63}$, Lina Wang ${ }^{63}$, Fuzhong Yang ${ }^{64}$, Enzhao Cong ${ }^{64}$, Jonathan Marchini ${ }^{1,65}$, Huanming Yang $^{3}$, Jian Wang ${ }^{3}$, Shenxun Shi ${ }^{64,66}$, Richard Mott ${ }^{1}$, Qi Xu ${ }^{67} \S$, Jun Wang ${ }^{3,68,69,70} \S$, Kenneth S. Kendler ${ }^{2} \S$ \& Jonathan Flint $^{1,71} \S$

${ }^{1}$ Wellcome Trust Centre for Human Genetics, University of Oxford, Roosevelt Drive, Oxford OX3 7BN, UK. ${ }^{2}$ Virginia Institute for Psychiatric and Behavioral Genetics, Virginia Commonwealth University, Richmond, Virginia 23298, USA. ${ }^{3}$ BGI-Shenzhen, Floor 9 Complex Building, Beishan Industrial Zone, YantianDistrict, Shenzhen, Guangdong 518083, China. ${ }^{4}$ Zhejiang Traditional Chinese Medical Hospital, No.54 Youdian Road, Hangzhou, Zhejiang 310000, China. ${ }^{5} \mathrm{CTSU}$, Richard Doll Building, University of Oxford, Old Road Campus, Oxford OX3 7LF, UK. ${ }^{6}$ Anhui Mental Health Center, No.316 Huangshan Road, Hefei, Anhui 230000, China. ${ }^{7}$ Anshan Psychiatric Rehabilitation Hospital, No.127 Shuangshan Road, Lishan District, Anshan, Liaoning 114000, China. ${ }^{8}$ Beijing Anding Hospital of Capital University of Medical Sciences, No.5 Ankang Hutong, Deshengmen wai, Xicheng District, Beijing, Beijing 100000 , China. ${ }^{9}$ Brain Hospital of Nanjing Medical University, No.264 Guangzhou Road, Nanjing, Jiangsu 210000, China. ${ }^{10}$ Changchun Mental Hospital, No.4596 Beihuan Road, Changchun, Jilin 130000 , China. ${ }^{11}$ Chongqing Mental Health Center, No.102 Jinzishan, Jiangbei District, Chongqing 404100, China.

${ }^{12}$ Dalian No.7 Hospital, No.179 Lingshui Road, Ganjingzi District, Dalian, Liaoning 116000 , China. ${ }^{13}$ Wuhan Mental Health Center, No.70, Youyi Road, Wuhan, Hubei 430000, China. ${ }^{14}$ Daqing No.3 Hospital of Heilongiiang Province, No.54 Xitai Road, Ranghulu district, Daqing, Heilongjiang 163000, China. ${ }^{15}$ First Hospital of Hebei Medical University, No.89 Donggang Road, Shijiazhuang, Hebei 50000, China. ${ }^{16}$ Fuzhou Psychiatric Hospital, No.451 South Erhuan Road, Cangshan District, Fuzhou, Fujian 350000, China. ${ }^{17}$ Guangxi Longquanshan Hospital, No.1 Jila Road, Yufeng District, Liuzhou, Guangxi Zhuangzu 545000, China. ${ }^{18}$ Guangzhou Brain Hospital, Guangzhou Psychiatric Hospital, No.36 Mingxin Road, Fangcun Avenue, Liwan District, Guangzhou, Guangdong 510000, China. ${ }^{19}$ Hainan Anning Hospital, No.10 East Nanhai Avenue, Haikou, Hainan 570100, China. ${ }^{20}$ Harbin Medical University, No.23 Youzheng street, Nangang District, Haerbin, Heilongjiang 150000, China. ${ }^{21}$ Harbin No.1 Special Hospital, No.217 Hongwei Road, Haerbin, Heilongjiang 150000, China. ${ }^{22}$ Hebei Mental Health Center, No.572 Dongfeng Road, Baoding, Hebei 71000, China. ${ }^{23}$ Huaian No.3 Hospital, No.272 West Huaihai Road, Huaian, Jiangsu 223001, China. ${ }^{24}$ Huzhou No.3 Hospital, No.255 Gongyuan Road, Huzhou, Zhejiang 313000, China. ${ }^{25}$ Jilin Brain Hospital, No.98 West Zhongyang Road, Siping, Jilin 136000, China. ${ }^{26}$ Jining Psychiatric Hospital, North Dai Zhuang, Rencheng District, Jining, Shandong 272000, China. ${ }^{27}$ Liaocheng No. 4 Hospital, No.47 North Huayuan Road, Liaocheng, Shandong 252000 , China. ${ }^{28}$ Mental Health Center of Shantou University, No.243 Daxue Road, Shantou, Guangdong 515000 China. ${ }^{29}$ Mental Health Center of West China Hospital of Sichuan University, No.28 South Dianxin Street, Wuhou District, Chengdu, Sichuan 610000, China. ${ }^{30}$ Mental Health
Institute of Jining Medical College, Dai Zhuang, Bei Jiao, Jining, Shandong 272000, China ${ }^{31}$ Mental Hospital of Jiangxi Province, No.43 Shangfang Road, Nanchang, Jiangxi 330000 , China. ${ }^{32}$ Mudanjiang Psychiatric Hospital of Heilongjiang Province, Xinglong, Mudanjiang, Heilongjiang 157000, China. ${ }^{33}$ Ningbo Kang Ning Hospital, No.1 Zhuangyu Road, Zhenhai District, Ningbo, Zhejiang 315000, China. ${ }^{34}$ No. 3 Hospital of Sun Yat-sen University, No.600 Tianhe Road, Tianhe District, Guangzhou, Guangdong 510630, China.

${ }^{35}$ No.1 Hospital of Chongqing Medical University, No.1 Youyi Road,Yuanjiagang,Yuzhong District, Chongqing, Chongqing 400016, China. ${ }^{36}$ No.1 Hospital of Jinan University, No.613 West Huangpu Avenue, Guangzhou, Guangdong 510000, China. ${ }^{37}$ No.1 Hospital of Medical College of Xian Jiaotong University, No. 277 West Yan Ta Road, Xian, Shaan Xi 710061, China. ${ }^{38}$ No.1 Hospital of Shanxi Medical University, No.85 South Jiefang Road, Taiyuan, Shanxi 30000, China. ${ }^{39}$ No. 1 Hospital of Zhengzhou University, No.1 East Jianshe Road, Zhengzhou, Henan 450000, China. ${ }^{40}$ No. 2 Hospital of Lanzhou University, No.82, Cuiyingmen, Lanzhou, Gansu 730000, China. ${ }^{41}$ No.2 Xiangya Hospital of Zhongnan University, No.139 Middle Renmin Road, Furong District, Changsha, Hunan 410000 , China. ${ }^{42}$ No.3 Hospital of Heilongiiang Province, No.135 Jiaotong Road, Beian, Heilongjiang 164000 , China. ${ }^{43}$ No.4 Hospital of Jiangsu University, No.246 Nanmen Street, Zhenjiang, Jiangsu 212000 , China. ${ }^{44}$ Psychiatric Hospital of Henan Province, No.388 Middle Jianshe Road, Xinxiang, Henan 453000, China. ${ }^{45}$ Qingdao Mental Health Center, No.299 Nanjing Road, Shibei District, Qingdao, Shandong 266000, China. ${ }^{46}$ Renmin Hospital of Wuhan University, No.238 Jiefang Road, Wuchang District, Wuhan, Hubei 430000 , China. ${ }^{47}$ Second Affiliated Hospital of Zhejiang Chinese Medical University, No.318 Chaowang Road, Hangzhou, Zhejiang 310000, China. ${ }^{48}$ ShengJing Hospital of China Medical University, No.36 Sanhao Street, Heping District, Shenyang, Liaoning 110001, China. ${ }^{49}$ Shenzhen Key Lab for Psychological Healthcare, Kangning Hospital, No.1080, Cuizhu Street, Luohu District, Shenzhen, Guangdong 518000, China. ${ }^{50}$ Department of General Internal Medicine, Kanazawa Medical University, Kahoku, Ishikawa 920-0293, Japan. ${ }^{51}$ Shenzhen Key Lab for Psychological Healthcare;Shenzhen Kangning Hospital, No.1080, Cuizhu Street, Luohu District, Shenzhen, Guangdong 518000, China. ${ }^{52}$ Sichuan Mental Health Center, No.190, East Jiannan Road, Mianyang, Sichuan 621000, China. ${ }^{53}$ Suzhou Guangji Hospital, No.286, Guangji Road, Suzhou, Jiangsu 215000, China. ${ }^{54}$ Tangshan No.5 Hospital, No.57 West Nanxin Road, Lunan District, Tangshan, Hebei 63000, China. ${ }^{55}$ The First Hospital of China Medical University, No.155 North Nanjing Street, Heping District, Shenyang, Liaoning 110001, China.

${ }^{56}$ Tianjin Anding Hospital, No.13 Liulin Road, Hexi District, Tianjin 300000, China. ${ }^{57}$ Tianjin First Center Hospital, No.55 Xuetang Street, Xinkai Road, Hedong District, Tianjin 300000, China. ${ }^{58}$ Tongji University Hospital, No.389 Xinchun Road, Shanghai 200000, China. ${ }^{59}$ Weihai Mental Health Center, Qilu Avenue, ETDZ, Weihai, Shandong 264200 China. ${ }^{60}$ Xian Mental Health Center, No.15 Yanyin Road, New Qujiang District, Xian, Shaanxi 710000, China. ${ }^{61}$ Xijing Hospital of No.4 Military Medical University, No.17 West Changle Road, Xian, Shaanxi 710000, China. ${ }^{62}$ Department of Comparative Developmental Genetics, Max Planck Institute for Plant Breeding Research, Carl-von-Linne-Weg 10, Cologne 50829, Germany. ${ }^{63}$ Shandong Mental Health Center, No.49 East Wenhua Road, Jinan, Shandong 250000, China. ${ }^{64}$ Shanghai Jiao Tong University School of Medicine, Shanghai Mental Health Centre, No. 600 Wan Ping Nan Road, Shanghai 200030, China. ${ }^{65}$ Department of Statistics, University of Oxford, Oxford OX1 3TG, UK. ${ }^{66}$ Fudan University affiliated Huashan Hospital, No. 12 Wulumuqi Zhong Road, Shanghai 200040, China. ${ }^{67}$ National Laboratory of Medical Molecular Biology, Institute of Basic Medical Sciences \& Neuroscience Center, Chinese Academy of Medical Sciences and Peking Union Medical College, Beijing 10005, China. ${ }^{68}$ Department of Biology, University of Copenhagen, Ole Maal Oes Vej 5, Copenhagen 2200, Denmark. ${ }^{69}$ Macau University of Science and Technology, Avenida Wai long, Taipa, Macau 999078 China, Taipa, Macau 999078, China. ${ }^{70}$ Princess Al Jawhara Center of Excellence in the Research of Hereditary Disorders, King Abdulaziz University, Jeddah 21589, Saudi Arabia. ${ }^{71}$ East China Normal University, 3663 North Zhongshan Road, Shanghai 200062, China.

*These authors contributed equally to this work. $\S$ These authors jointly supervised this work. 


\section{METHODS}

No statistical methods were used to predetermine sample size.

Sample collection. CONVERGE collected cases of recurrent major depression from 58 provincial mental health centres and psychiatric departments of general medical hospitals in 45 cities and 23 provinces of China. Controls were recruited from patients undergoing minor surgical procedures at general hospitals (37\%) or from local community centres (63\%). A sample size of 6,000 cases and 6,000 controls was chosen on the basis of evidence available when the study was designed (in 2007) of the likely existence of genetic loci with odds ratio of 1.2 and above. All subjects were Han Chinese women with four Han Chinese grandparents. Cases were excluded if they had a pre-existing history of bipolar disorder, psychosis or mental retardation. Cases were aged between 30 and 60 and had two or more episodes of MDD meeting DSM-IV criteria ${ }^{21}$ with the first episode occurring between 14 and 50 years of age, and had not abused drugs or alcohol before their first depressive episode. All subjects were interviewed using a computerized assessment system. Interviewers were postgraduate medical students, junior psychiatrists or senior nurses, trained by the CONVERGE team for a minimum of 1 week. The diagnosis of MDD was established with the Composite International Diagnostic Interview (CIDI) (WHO lifetime version 2.1; Chinese version), which used DSM-IV criteria. The interview was originally translated into Mandarin by a team of psychiatrists at Shanghai Mental Health Centre, with the translation reviewed and modified by members of the CONVERGE team.

The replication sample was obtained from five hospitals in the north of China. Patients were diagnosed as having MDD by at least two consultant psychiatrists by DSM-IV criteria. Samples were of both sexes, and all four grandparents were Han Chinese. Cases were aged between 30 and 60, and had two or more episodes of MDD meeting DSM-IV criteria. Exclusion criteria were pregnancy, severe medica conditions, abnormal laboratory baseline values, unstable psychiatric features (for example, suicidal), a history of alcoholism or drug abuse, epilepsy, brain trauma with loss of consciousness, neurological illness, or a concomitant axis I psychiatric disorder. Control subjects were recruited from local communities and provided information about medical and family histories. Exclusion criteria were a history of major psychiatric or neurological disorders, psychiatric treatment or drug abuse, or a family history of severe forms of psychiatric disorders.

The study protocol was approved centrally by the Ethical Review Board of Oxford University (Oxford Tropical Research Ethics Committee) and the ethics committees of all participating hospitals in China. All interviewers were mental health professionals who are well able to judge decisional capacity. The study posed minimal risk (an interview and saliva sample). All participants provided their written informed consent.

DNA sequencing. DNA was extracted from saliva samples using the Oragene protocol. A barcoded library was constructed for each sample. All saliva samples were randomized in allocation to sequencing batches, and experimenters performing the sequencing procedure were blinded to sample allocation and outcome assessment. Sequencing reads obtained from Illumina Hiseq machines were aligned to Genome Reference Consortium Human Build 37 patch release 5 (GRCh37.p5) with Stampy (v1.0.17) 22 using default parameters after filtering out reads containing adaptor sequences or consisting of more than $50 \%$ poor quality (base quality $\leq 5)$ bases. Samtools $(\mathrm{v} 0.1 .18)^{23}$ was used to index the alignments in BAM format ${ }^{23}$, and Picardtools (v1.62) was used to mark PCR duplicates for downstream filtering. The Genome Analysis Toolkit's (GATK, version 2.6) ${ }^{24}$ BaseRecalibrator was then run on the BAM files to create base quality score recalibration tables, masking known SNPs and INDELs from dbSNP (version 137, excluding all sites added after version 129). Base quality recalibration (BQSR) was then performed on the BAM files using GATKlite (v2.2.15) ${ }^{24}$ while also removing read pairs that did not have the 'properly aligned segment' bit set by Stampy ( $1-5 \%$ of reads per sample).

Variant calling. Variant discovery and genotyping at all polymorphic SNPs in the 1000G Phasel East Asian (ASN) reference panel ${ }^{14}$ was performed simultaneously using post-BQSR sequencing reads from all samples using the GATK's UnifiedGenotyper (version 2.7-2-g6bda569). We set the option '--genotype likelihood model' to 'BOTH', used default annotation outputs for variant calls, and set the '--dbSNP' option in order to use dbSNP v137 rsids to fill in the variant ID column of the output variant call format (VCF) files. Variant quality score recalibration was then performed on these sites using the GATK's VariantRecalibrator (version 2.7-2-g6bda569) and the biallelic SNPs from 1000G Phase1 ASN samples as a true positive set of variants. A sensitivity threshold of $90 \%$ to SNPs in the 1000G Phase1 ASN panel was applied for SNP selection for imputation after optimizing for Transition to Transversion (TiTv) ratios in SNPs called. This gave a total of 21,356,798 (9,053,391 known in 1000 Genomes Phase 1 ASN Panel and 11,486,024 novel) biallelic SNPs identified from all chromosomes and unassembled contigs. We put forth 20,539,441 SNPs from the autosomes and chromosome $\mathrm{X}$ for imputation of genotype probabilities and downstream analyses.

Genotype likelihood calculation and imputation. Genotype likelihoods (GLs) were calculated at all 20,539,441 SNPs using a sample-specific binomial mixture model implemented in SNPtools (version 1.0) (25 $^{25}$ and imputation was performed

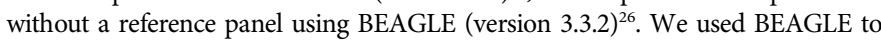
perform imputation, using ten iterations on chunks of 3,000 SNPs with 600 SNPs of overlap. A second round of imputation was performed with BEAGLE on the same GLs, but only at biallelic SNPs polymorphic in the 1000G Phase 1 ASN panel using 572 haplotypes from the 1000 Genomes Phase 1 ASN samples as a reference panel for six iterations on chunks containing roughly 3,000 SNPs with 600 SNPs of overlap. After both rounds of imputation we removed the outer 300 SNPs of every window and ligated imputation results of adjacent chunks. A final set of allele dosages and genotype probabilities was generated from these two sets of imputed results by replacing the results in the former with those in the latter at all sites imputed in the latter. We then applied a conservative set of inclusion thresholds for SNPs for GWAS: (a) $P$ value for violation of the Hardy-Weinberg equilibrium $>10^{-6}$; (b) information score $>0.9$; (c) MAF in CONVERGE $>0.5 \%$, to arrive at the final set of 6,242,619 SNPs for GWAS.

Sample selection for GWAS. Using both processed sequencing data and imputed dosages at SNPs that passed quality control, we assessed the sequencing and imputation quality of all 11,670 samples whose genomic variants we imputed. We first looked into both the nuclear genome and mitochondrial genome for an excess of variants called, since this would indicate cross-sample contamination due to technical issues during sequencing. We quantified the number of singleton variants called in genic regions of the nuclear genome and found a mean of 71.55 private variants per sample that were supported by more than 2 sequencing reads passing sequencing quality controls. We excluded 117 samples with a number of singletons greater than the $99^{\text {th }}$ percentile. Coverage of the mitochondrial genome was, on average, $102 \times$, allowing us to obtain high-quality sequences for this part of the genome. We found a mean of 15.70 heteroplasmic sites per sample, and 116 samples were found to have greater than the 99th percentile of the number of heteroplasmic sites. Of these 116 samples, 26 were already discarded for having excess nuclear genome singletons; and we excluded the remaining 90 .

We then checked imputation quality based on the certainty of genotypes imputed (maximum genotype probability $>0.9$ ). We identified 29 individuals who had fewer than $90 \%$ of their sites with maximum genotype probabilities $>0.9$. We excluded these samples from further analysis.

Finally, we assessed the 11,434 remaining samples for genetic relatedness. Although being unrelated to other individuals recruited for the CONVERGE study was a clear criteria in our data collection process, there were instances when the same patient or a relative of the patient visited multiple hospitals and was thus recruited more than once. To exclude duplicates and first-degree relatives from our sample for GWAS, we estimated pairwise genome-wide identity by descent (IBD) using identity by state (IBS) information in hard-called genotypes from imputed genotype probabilities at 399,211 common tagging SNPs across all autosomes (MAF $>1 \%$, linkage disequilibrium $(\mathrm{LD})<0.5$, all known in 1000 Genomes Phase 1). We implemented this in PLINK (v1.07) ${ }^{27}$ with the option '--genome'. We excluded a total of 392 samples (duplicates and first-degree relatives) from our final set of samples for GWAS. We retained second-degree relatives and beyond, correcting for the relatedness between them using a linear mixed model. We also excluded 402 samples with incomplete phenotype information, giving a final set of 10,640 samples (5,303 cases of MDD, 5,337 controls) for the primary GWAS of MDD.

GWAS using linear mixed model and liability score estimates. We implemented MLMA using factored spectrally transformed linear mixed models (FastLMM v2.06.20130802 $)^{9,10}$ and computed one GRM per chromosome using the mixed linear model with candidate marker excluded (MLMe) approach, removing the SNPs from the chromosome in question from a base set of 322,911 common tagging SNPs from all autosomes $(\mathrm{MAF}>1 \%, \mathrm{LD}<0.5$, all known in $1000 \mathrm{G}$ Phasel ASN panel) to prevent loss of power through 'double fitting' of the candidate SNP (and those in LD with it) in the GRM as a random effect, while testing each SNP as a fixed effect. Manhattan plots and quantile-quantile plots of the $\log _{10}$ of $P$ values of the GWAS were generated with custom code in R (ref. 28). Genomic control inflation factor $\lambda$ was calculated using custom code in R (ref. 28).

Replication and joint analyses. We genotyped the replication sample on a MassARRAY system mass spectrometer. TYPER4.0 was used to assess the reliability of genotype calls generated by SpectroREAD from the mass spectra. Default genotype call inclusion criteria were used. To perform the association analysis with MDD case-control status at these 12 sites in the replication sample, we obtained effect sizes for discovery from logistic regression with principal component (PC) correction, and then for replication from logistic regression, and then performed fixed-effects meta-analysis. 
Polygenic risk profiling and binomial sign-test. Single SNP association results were obtained from the PGC study of $\mathrm{MDD}^{3}$. Prior to analysis, SNPs were lifted over to GRCh37/hg19 coordinates and excluded if: (a) monomorphic in either European $(n=379)$ or East Asian $(n=286)$ populations from the 1000 Genomes Project Phase 1 reference data ${ }^{14}$; or (b) absent from the filtered CONVERGE data set. To construct the PGC-trained polygenic score, we initially selected autosomal SNPs with statistical imputation information (information score) greater than 0.9 and MAF greater than $1 \%$ in both studies, and performed subsequent LD-based 'clumping' to remove markers from highly correlated SNP pairs (pairwise $r^{2}>0.2$ in East Asians, $500 \mathrm{~kb}$ window) while preferentially retaining SNPs with smaller PGC $P$ values. Using the resultant SNP set, we constructed polygene scores based on varying $P$ value thresholds $\left(1 \times 10^{-6}, 1 \times 10^{-5}, 1 \times 10^{-4}, 1 \times 10^{-3}, 0.01,0.1,0.2,0.3,0.4,0.5\right.$, and 1$)$ as previously described ${ }^{29}$. We assessed the predictive value of polygenic scores in a genetically unrelated subset of the CONVERGE sample (with pairwise relatedness less than 0.1) by logistic regression, with adjustment for ancestry principal components, demonstrating significant association with MDD status. The estimated variance in MDD risk accounted for by the polygenic score is given by Nagelkerke's $R^{2}$. Using the same $P$ value thresholds, we tabulated the number of independent SNPs with the same direction of allelic effect in the PGC results as observed in CONVERGE. The filtering criteria for SNPs was an information score greater than 0.9 in CONVERGE and MAF greater than $1 \%$ in both studies; and an analogous LD-clumping procedure was performed (pairwise $r^{2}>0.2$ in Europeans, $500 \mathrm{~kb}$ window). A one-sided binomial sign test was used to assess whether this observed fraction was significantly greater than that expected by chance. Results are given in Extended Data Table 4.

21. Association, A. P. Diagnostic and statistical manual of mental disorders 4th edn (American Psychiatric Association, 1994).

22. Lunter, G. \& Goodson, M. Stampy: a statistical algorithm for sensitive and fast mapping of Illumina sequence reads. Genome Res. 21, 936-939 (2011).

23. Li, H. et al. The Sequence Alignment/Map format and SAMtools. Bioinformatics $\mathbf{2 5}$ 2078-2079 (2009).

24. McKenna, A. et al. The Genome Analysis Toolkit: a MapReduce framework for analyzing next-generation DNA sequencing data. Genome Res. 20, 1297-1303 (2010).

25. Wang, Y. et al. An integrative variant analysis pipeline for accurate genotype/ haplotype inference in population NGS data. Genome Res. 23, 833-842 (2013).

26. Browning, S. R. \& Browning, B. L. Rapid and accurate haplotype phasing and missing-data inference for whole-genome association studies by use of localized haplotype clustering. Am. J. Hum. Genet. 81, 1084-1097 (2007).

27. Purcell, S. et al. PLINK: a tool set for whole-genome association and populationbased linkage analyses. Am. J. Hum. Genet. 81, 559-575 (2007).

28. R Development Core Team. A language and environment for statistical computing ( $R$ Foundation for Statistical Computing, 2004).

29. Dudbridge, F. Power and predictive accuracy of polygenic risk scores. PLoS Genet 9, e1003348 (2013) 


\section{RESEARCH LETTER}

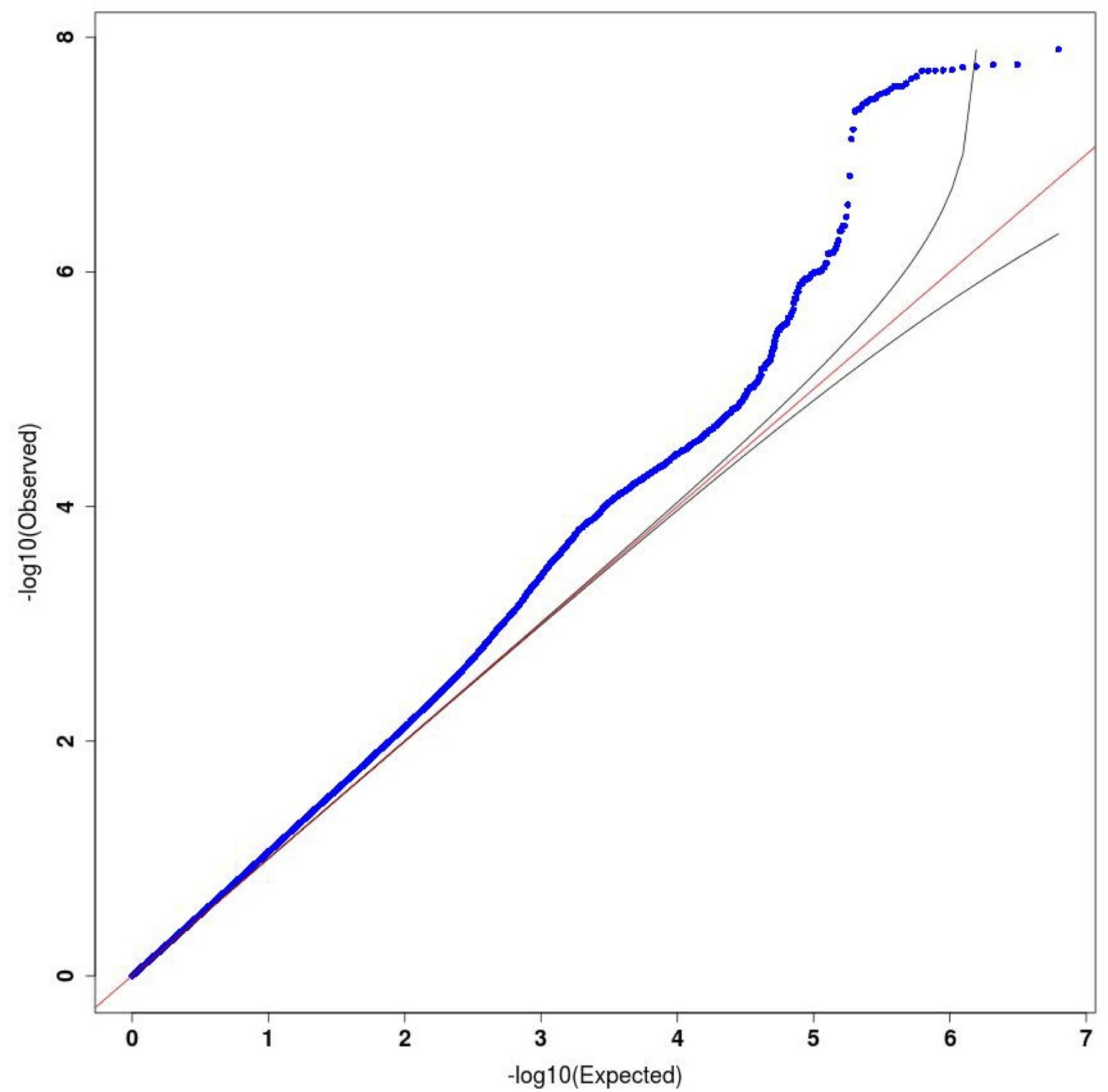

Extended Data Figure $1 \mid$ Quantile-quantile plots for major depressive disorder. Quantile-quantile plot of GWAS for MDD using the mixed linear model with exclusion of the chromosome that the marker is on (MLMe) method implemented in FastLMM on 10,640 samples (5,303 cases, 5,337 controls). Genomic inflation factor $\lambda=1.070$, rescaled for an equivalent study of 1,000 cases and 1,000 controls $\left(\lambda_{1000}\right)=1.013$. 

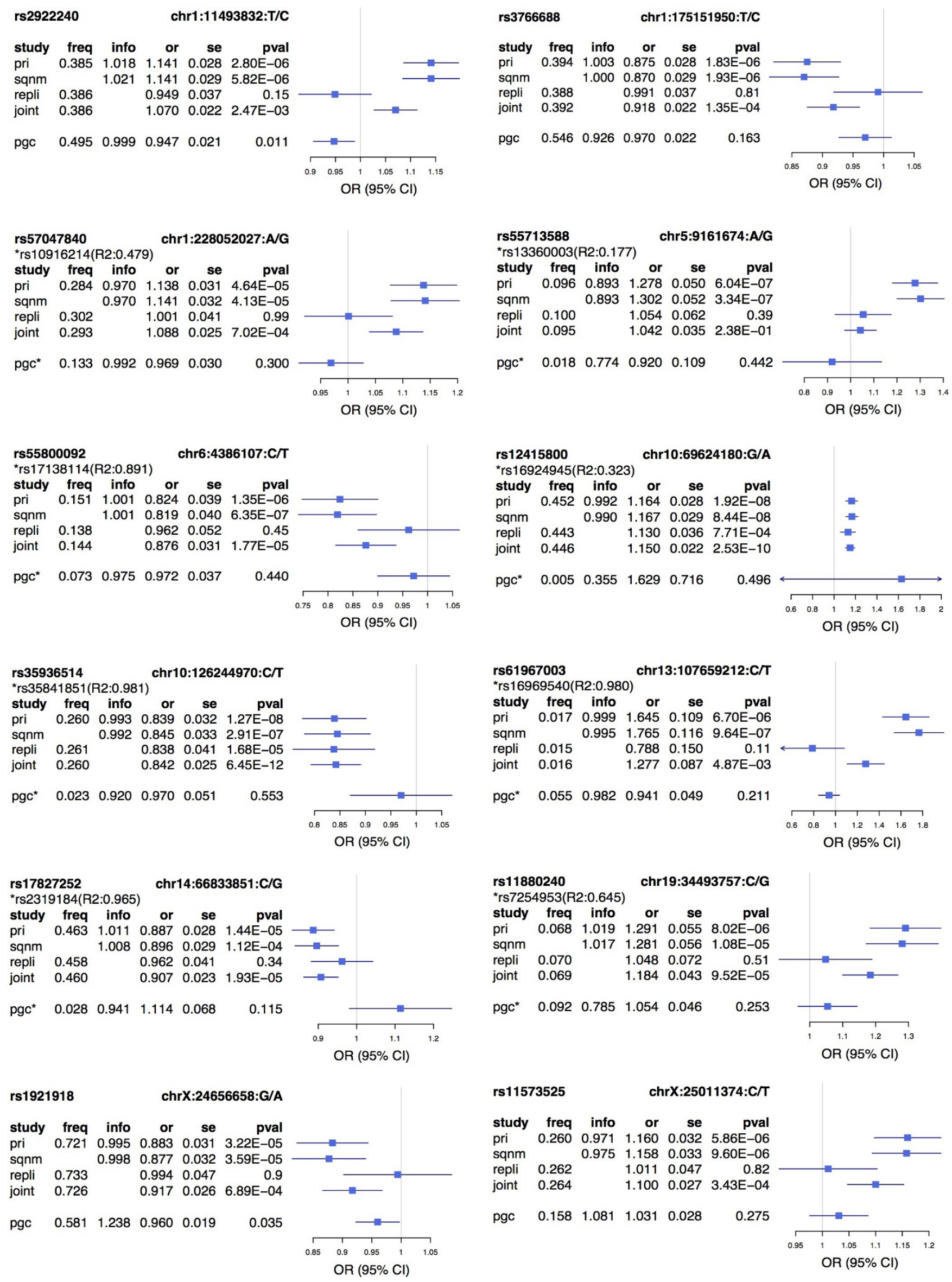

\section{rs11573525 chrX:25011374:C/T}

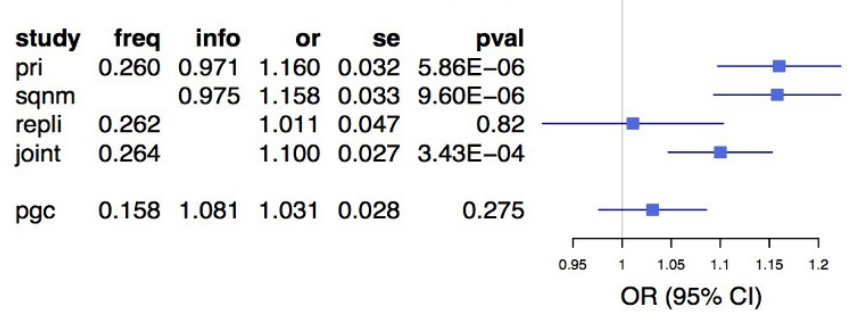




\section{RESEARCH LETTER}

Extended Data Figure $2 \mid$ Forest plots of estimated SNP effects in CONVERGE and PGC studies. This figure presents the association odds ratios (OR) at 12 SNPs in CONVERGE and the best available proxy SNPs in PGC-MDD (pairwise $r^{2}>0.6,500 \mathrm{~kb}$ window; the proxy SNP is marked by an asterisk). We present the alternative allele frequency (freq), odds ratio (or) with respect to the alternative allele, standard error of odds ratio (se) and $P$ values of association (pval) for the following analyses (study): primary association analysis with a linear-mixed model using imputed allele dosages in 10,640 samples in CONVERGE (pri); validation analysis with logistic regression model with principal components (PCs) as covariates using genotypes from Sequenom on 9,921 samples in CONVERGE (sqnm); association with MDD with a logistic regression model in a replication cohort of 6,417 samples using genotypes from Sequenom (repli); joint association analysis with MDD with a logistic regression model using imputed allele dosages in CONVERGE and genotypes from Sequenom in a replication cohort (17,057 samples in total; joint). 
a

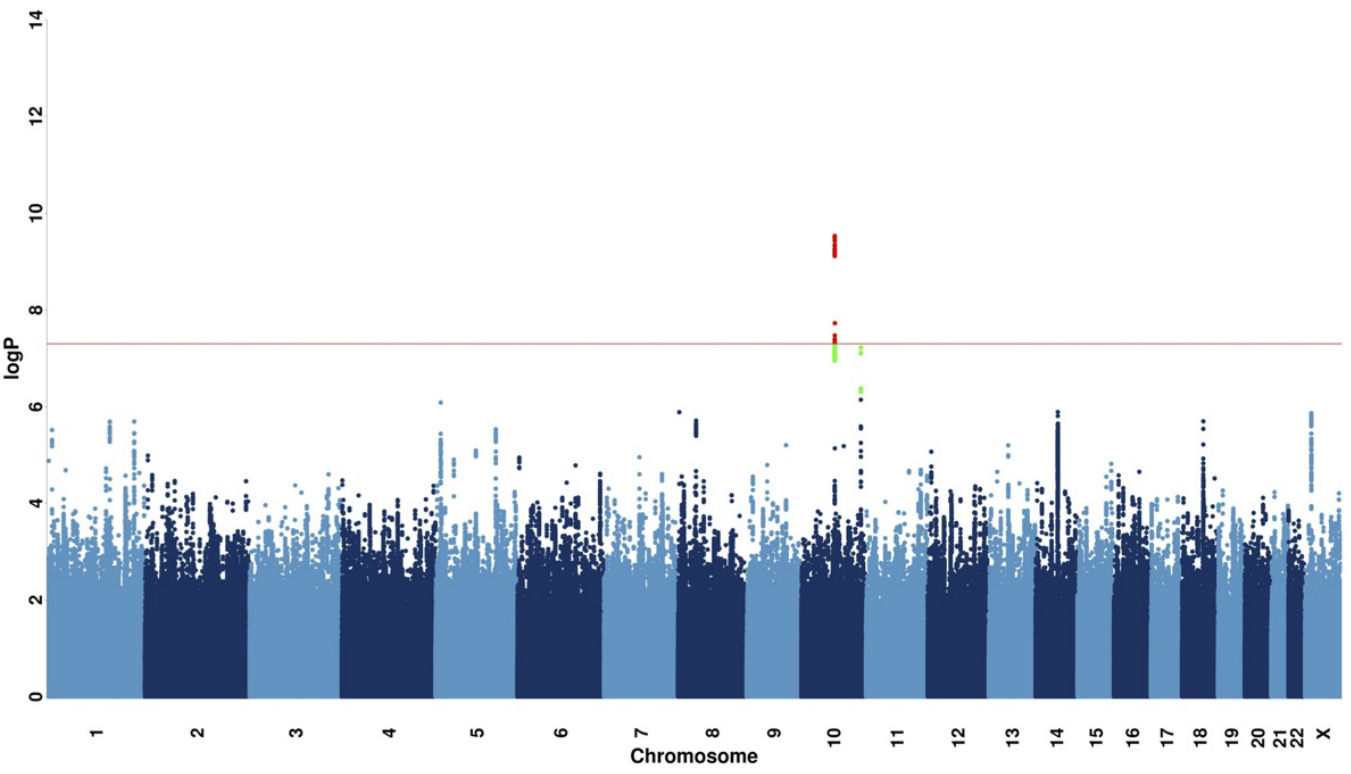

b

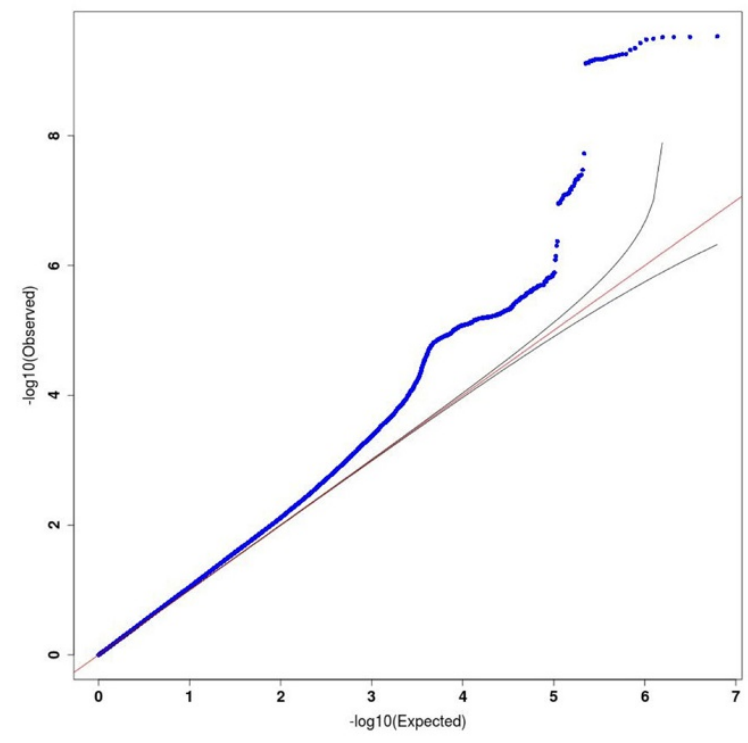

c

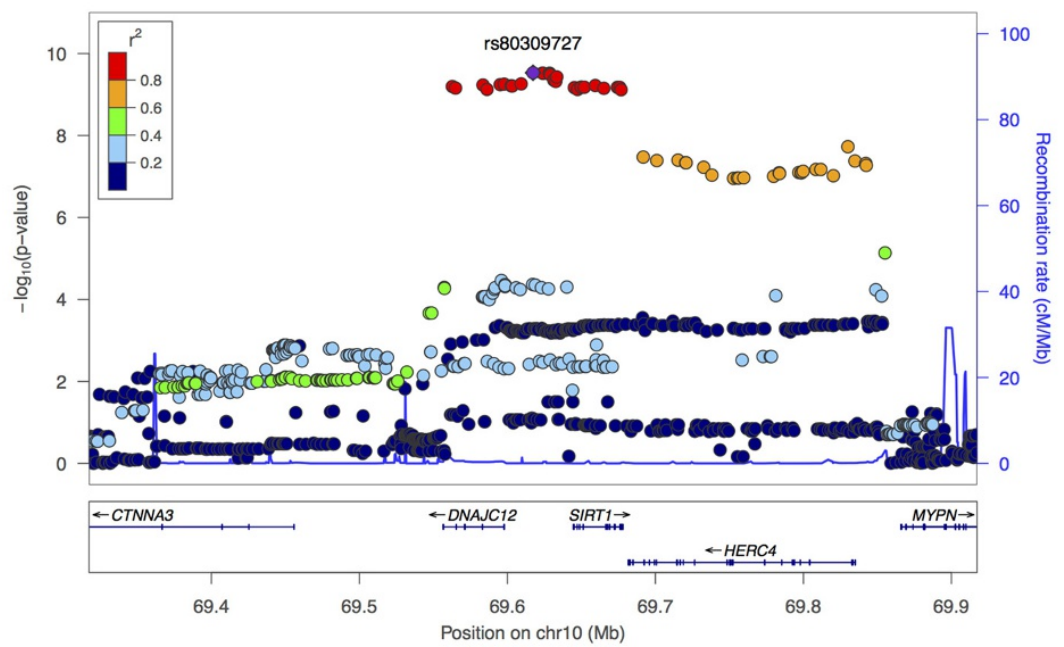

Extended Data Figure $3 \mid$ Manhattan and quantile quantile plots for melancholia. a, Manhattan plot of GWAS for melancholia using the MLMe method implemented in FastLMM on 9,846 samples (4,509 cases, 5,337 controls). b, Quantile-quantile plot of GWAS for melancholia; $\lambda=1.069$, $\lambda_{1000}=1.014$. c, Regional association plot of GWAS hits on chromosome 10, focusing on top SNP rs80309727 at 5' of SIRT1 gene, generated with LocusZoom. 


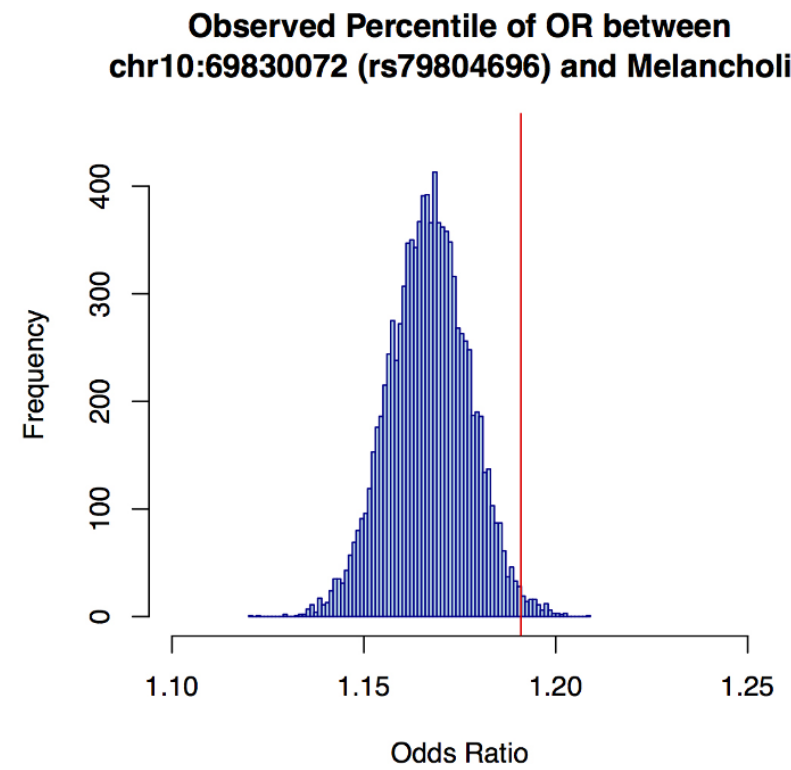

Extended Data Figure $4 \mid$ Empirical estimation of the odds ratio increases due to the removal of cases not falling under the diagnostic class of melancholia from an association analysis with major depression. The figures show the empirical distributions of the odds ratios for association with each of two SNPs (rs79804696, rs35936514), after removing a random set of 796

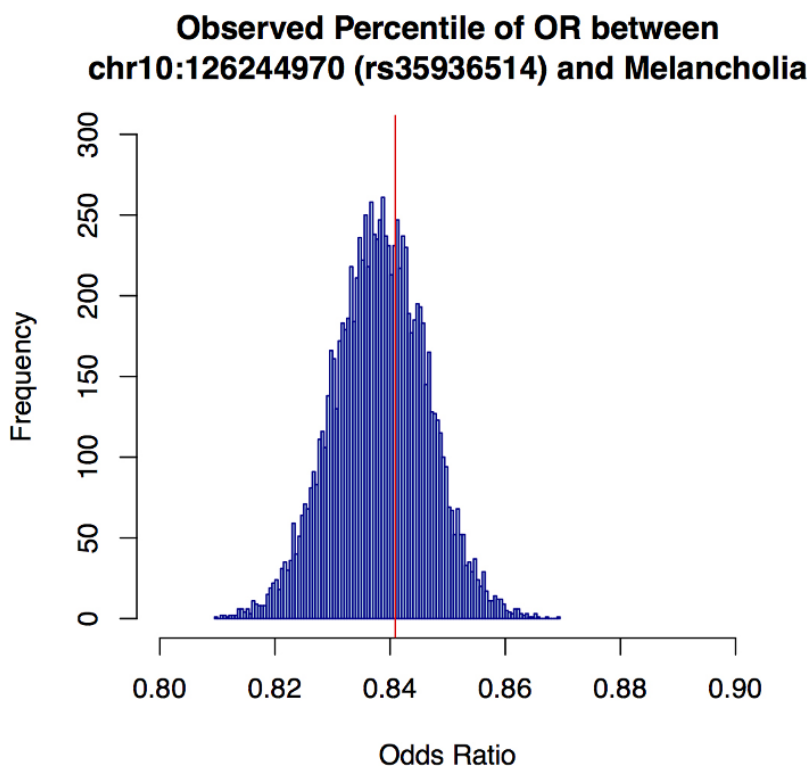

samples, equal to the number of cases of MDD not diagnosed as being melancholic. The horizontal axis is the odds ratio for each analysis, and the vertical axis the frequency of occurrence of the odds ratio in 10,000 analyses. The vertical red line is the observed odds ratio after removing cases of MDD not diagnosed as melancholic. 
Extended Data Table 1 | Comparison between association results using imputed dosages and directly genotyped markers

\begin{tabular}{|c|c|c|c|c|c|c|c|c|c|c|c|c|}
\hline \multicolumn{4}{|c|}{ SNP } & \multicolumn{5}{|c|}{ Imputed Dosages $(\mathrm{N}=9,921)$} & \multicolumn{4}{|c|}{ Sequenom genotypes } \\
\hline CHR & POS & RSID & REF & ALT & OR & SE & $\mathbf{P}$ & $\mathbf{N}$ & $r^{2}$ & OR & SE & $\mathbf{P}$ \\
\hline 1 & 11493832 & rs2922240 & $\mathrm{C}$ & $T$ & 1.141 & 0.029 & $5.82 E-06$ & 9,864 & 0.991 & 1.141 & 0.029 & 5.72E-06 \\
\hline 1 & 175151950 & rs3766688 & $\mathrm{C}$ & $T$ & 0.870 & 0.029 & $1.93 \mathrm{E}-06$ & 9,901 & 0.995 & 0.871 & 0.029 & 2.32E-06 \\
\hline 1 & 228052027 & rs57047840 & G & A & 1.141 & 0.032 & 4.13E-05 & 9,724 & 0.974 & 1.141 & 0.032 & 3.91E-05 \\
\hline 5 & 9161674 & rs55713588 & G & A & 1.302 & 0.052 & 3.34E-07 & 9,636 & 0.925 & 1.263 & 0.050 & 2.87E-06 \\
\hline 6 & 4386107 & rs 55800092 & $\mathrm{~T}$ & C & 0.819 & 0.040 & $6.35 \mathrm{E}-07$ & 9,881 & 0.992 & 0.817 & 0.040 & $5.52 \mathrm{E}-07$ \\
\hline 10 & 69624180 & rs12415800 & A & G & 1.167 & 0.029 & $8.44 \mathrm{E}-08$ & 9,689 & 0.993 & 1.167 & 0.029 & $9.30 \mathrm{E}-08$ \\
\hline 10 & 126244970 & rs35936514 & $T$ & C & 0.845 & 0.033 & 2.91E-07 & 9,915 & 0.993 & 0.842 & 0.033 & $1.40 \mathrm{E}-07$ \\
\hline 13 & 107659212 & rs61967003 & $T$ & C & 1.765 & 0.116 & $9.64 \mathrm{E}-07$ & 9,914 & 0.997 & 1.748 & 0.116 & $1.53 \mathrm{E}-06$ \\
\hline 14 & 66833851 & rs 17827252 & $\mathrm{G}$ & C & 0.896 & 0.029 & $1.12 \mathrm{E}-04$ & 8,562 & 0.999 & 0.897 & 0.031 & 3.94E-04 \\
\hline 19 & 34493757 & rs 11880240 & G & C & 1.281 & 0.056 & $1.08 \mathrm{E}-05$ & 9,912 & 0.996 & 1.281 & 0.056 & $1.14 \mathrm{E}-05$ \\
\hline$x$ & 24656658 & rs1921918 & A & G & 0.877 & 0.032 & $3.59 \mathrm{E}-05$ & 9,899 & 0.994 & 0.880 & 0.032 & $6.39 \mathrm{E}-05$ \\
\hline$x$ & 25011374 & rs 11573525 & T & C & 1.158 & 0.033 & $9.60 \mathrm{E}-06$ & 9,912 & 0.969 & 1.144 & 0.032 & $3.21 \mathrm{E}-05$ \\
\hline
\end{tabular}

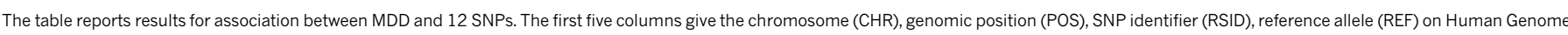

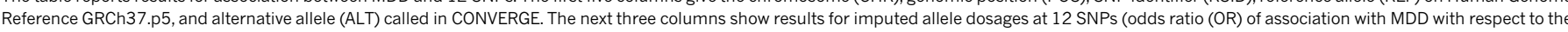

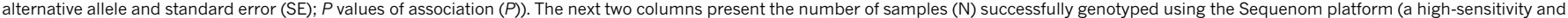

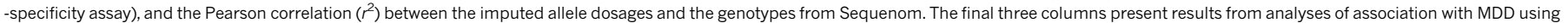
genotypes from the Sequenom genotyping platform. Bold type indicates the genome-wide significant markers; Extended Data Table 2 gives further information on the results for these markers. 


\section{RESEARCH LETTER}

Extended Data Table 2 | Genotype distribution and $P$ values for violation of the Hardy-Weinberg equilibrium in CONVERGE and replication cohorts

\begin{tabular}{llllll}
\hline \multirow{2}{*}{$\begin{array}{c}\text { MDD } \\
\text { Disease State }\end{array}$} & \multirow{2}{*}{ SNP } & \multicolumn{2}{c}{ CONVERGE } & \multicolumn{2}{c}{ Replication Cohort } \\
\cline { 3 - 6 } & & HomRef/Het/HomAlt & HWE P-value & HomRef/Het/HomAlt & HWE P-value \\
\hline \multirow{2}{*}{ All } & rs12415800 & $2151 / 5301 / 3169$ & 0.445 & $1212 / 3037 / 1920$ & 0.857 \\
& rs35936514 & $705 / 4054 / 5794$ & 0.919 & $422 / 2400 / 3398$ & 0.974 \\
Cases & rs12415800 & $1178 / 2626 / 1490$ & 0.741 & $654 / 1538 / 918$ & 0.829 \\
& rs35936514 & $318 / 1919 / 3027$ & 0.549 & $190 / 1136 / 1783$ & 0.627 \\
Controls & rs12415800 & $973 / 2675 / 1679$ & 0.106 & $558 / 1499 / 1002$ & 0.971 \\
& rs35936514 & $387 / 2135 / 2767$ & 0.389 & $232 / 1264 / 1615$ & 0.503 \\
\hline
\end{tabular}

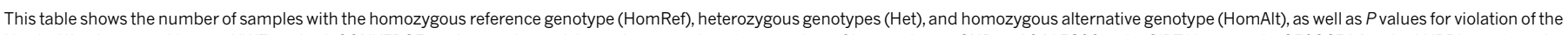

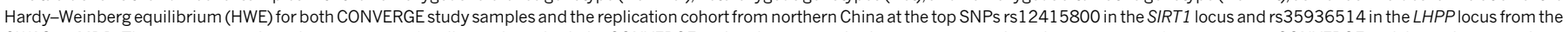

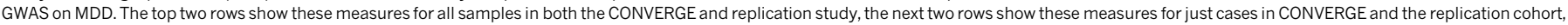

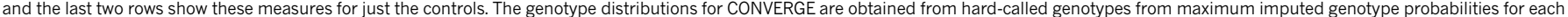

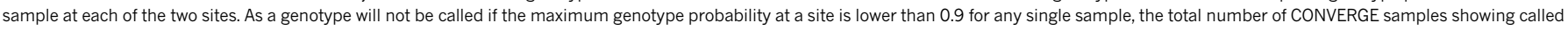

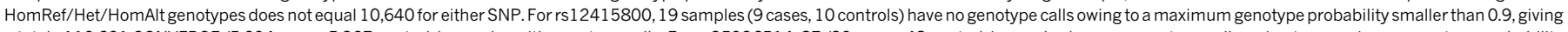

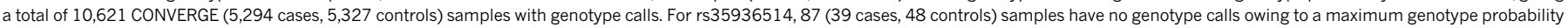
smaller than 0.9 , giving a total of 10,553 (5,264 cases, 5,289 controls) CONVERGE samples with genotype calls. 
Extended Data Table 3 | Single-marker association results of top CONVERGE hits in the PGC study of MDD

\begin{tabular}{|c|c|c|c|c|c|c|c|c|c|c|c|c|c|c|}
\hline \multicolumn{9}{|c|}{ CONVERGE ( 10,640 samples) } & \multicolumn{6}{|c|}{ PGC MDD (18,759 samples) } \\
\hline CHR & POS & RSID & REF & ALT & FREQ & OR & SE & $\mathbf{P}$ & RSID & LD r2 & FREQ & INFO & OR & $\mathbf{P}$ \\
\hline 1 & 11493832 & rs2922240 & C & $\mathrm{T}$ & 0.3846 & 1.141 & 0.028 & $2.80 \mathrm{E}-06$ & rs2922240 & 1.00 & 0.495 & 0.999 & 0.947 & 0.011 \\
\hline 1 & 175151950 & rs3766688 & C & $\mathrm{T}$ & 0.394 & 0.875 & 0.028 & $1.83 \mathrm{E}-06$ & rs3766688 & 1.00 & 0.546 & 0.926 & 0.970 & 0.163 \\
\hline 1 & 228052027 & rs57047840 & G & A & 0.2843 & 1.138 & 0.031 & 4.64E-05 & rs10916214 & 0.48 & 0.133 & 0.992 & 0.969 & 0.300 \\
\hline 5 & 9161674 & rs55713588 & G & A & 0.0956 & 1.278 & 0.050 & $6.04 \mathrm{E}-07$ & rs13360003 & 0.18 & 0.018 & 0.774 & 0.920 & 0.442 \\
\hline 6 & 4386107 & rs55800092 & $T$ & C & 0.1512 & 0.824 & 0.039 & $1.35 \mathrm{E}-06$ & rs17138114 & 0.89 & 0.073 & 0.975 & 0.972 & 0.440 \\
\hline 10 & 69624180 & rs 12415800 & A & $\mathbf{G}$ & 0.4519 & 1.164 & 0.028 & $1.92 \mathrm{E}-08$ & rs16924945 & 0.32 & 0.005 & 0.355 & 1.629 & 0.496 \\
\hline 10 & 126244970 & rs35936514 & $\mathbf{T}$ & C & 0.2609 & 0.839 & 0.032 & $1.27 \mathrm{E}-08$ & rs35841851 & 0.98 & 0.023 & 0.92 & 0.970 & 0.553 \\
\hline 13 & 107659212 & rs61967003 & $T$ & C & 0.0172 & 1.645 & 0.109 & $6.70 \mathrm{E}-06$ & rs16969540 & 0.98 & 0.055 & 0.982 & 0.941 & 0.211 \\
\hline 14 & 66833851 & rs 17827252 & $\mathrm{G}$ & C & 0.4624 & 0.887 & 0.028 & $1.44 \mathrm{E}-05$ & rs2319184 & 0.96 & 0.028 & 0.941 & 1.114 & 0.115 \\
\hline 19 & 34493757 & rs 11880240 & $\mathrm{G}$ & C & 0.0679 & 1.291 & 0.055 & 8.02E-06 & rs7254953 & 0.65 & 0.092 & 0.785 & 1.054 & 0.253 \\
\hline$x$ & 24656658 & rs1921918 & A & $\mathrm{G}$ & 0.7206 & 0.883 & 0.031 & 3.22E-05 & rs1921918 & 1.00 & 0.581 & 1.238 & 0.960 & 0.035 \\
\hline$x$ & 25011374 & rs 11573525 & $\mathrm{~T}$ & C & 0.2602 & 1.160 & 0.032 & $5.86 \mathrm{E}-06$ & rs 11573525 & 1.00 & 0.158 & 1.081 & 1.031 & 0.275 \\
\hline
\end{tabular}

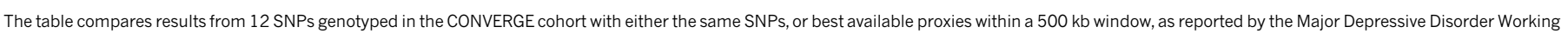

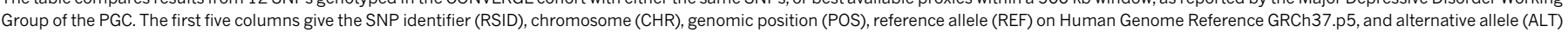

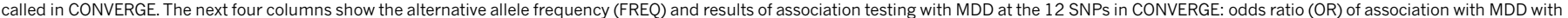

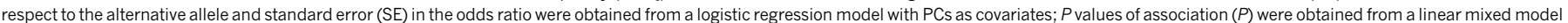

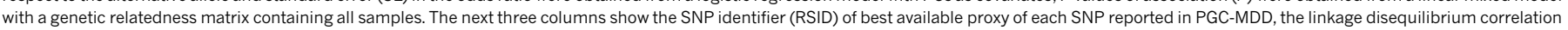

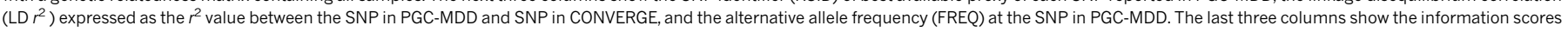
(INFO), odds ratios (OR) and $P$ values of association with MDD in PGC-MDD from a logistic regression model. Bold type indicates the genome-wide significant markers. 


\section{RESEARCH LETTER}

Extended Data Table 4 | Polygenic risk profiling and binomial sign tests

\begin{tabular}{lllll}
\hline \multirow{2}{*}{$\boldsymbol{p} T$} & \multicolumn{3}{c}{ Polygenic risk profiling } & \multicolumn{2}{c}{ Binomial sign test } \\
\cline { 2 - 5 } & & $\boldsymbol{r}^{2}$ & $\boldsymbol{P}$ & \multicolumn{1}{c}{ No. SNPs (\%) } \\
\hline 0.000001 & 0.000715 & $\mathbf{0 . 0 1 7 4}$ & $3(100)$ & 0.125 \\
0.00001 & $8.40 \mathrm{E}-05$ & 0.415 & $12(66.7)$ & 0.194 \\
0.0001 & $2.57 \mathrm{E}-05$ & 0.652 & $62(58.1)$ & 0.126 \\
0.001 & $5.87 \mathrm{E}-06$ & 0.829 & $481(53.6)$ & 0.0605 \\
0.01 & $8.67 \mathrm{E}-05$ & 0.407 & $3632(51.1)$ & 0.101 \\
0.1 & 0.00142 & 0.000797 & $26106(50.4)$ & 0.126 \\
0.2 & 0.00126 & 0.00156 & $45166(50.6)$ & 0.00331 \\
0.3 & 0.00116 & 0.00246 & $61074(50.5)$ & 0.00627 \\
0.4 & 0.00125 & 0.00168 & $74676(50.5)$ & 0.00335 \\
0.5 & 0.0011 & 0.00317 & $86429(50.4)$ & 0.00758 \\
1 & 0.000924 & 0.00684 & $124361(50.3)$ & 0.0116 \\
\hline
\end{tabular}

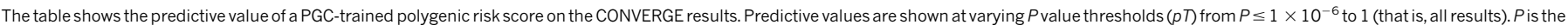

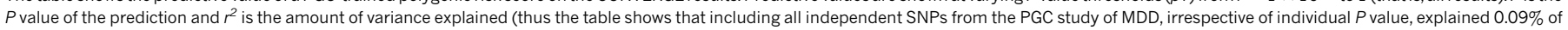

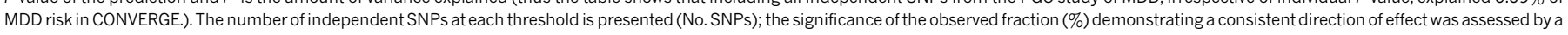
one-sided binomial sign test. 